\title{
¿COLOMBIA RESPONDE A LAS EXIGENCIAS DE LA CALIDAD EN LA EDUCACIÓN SUPERIOR COMO PILAR DE CRECIMIENTO?
}

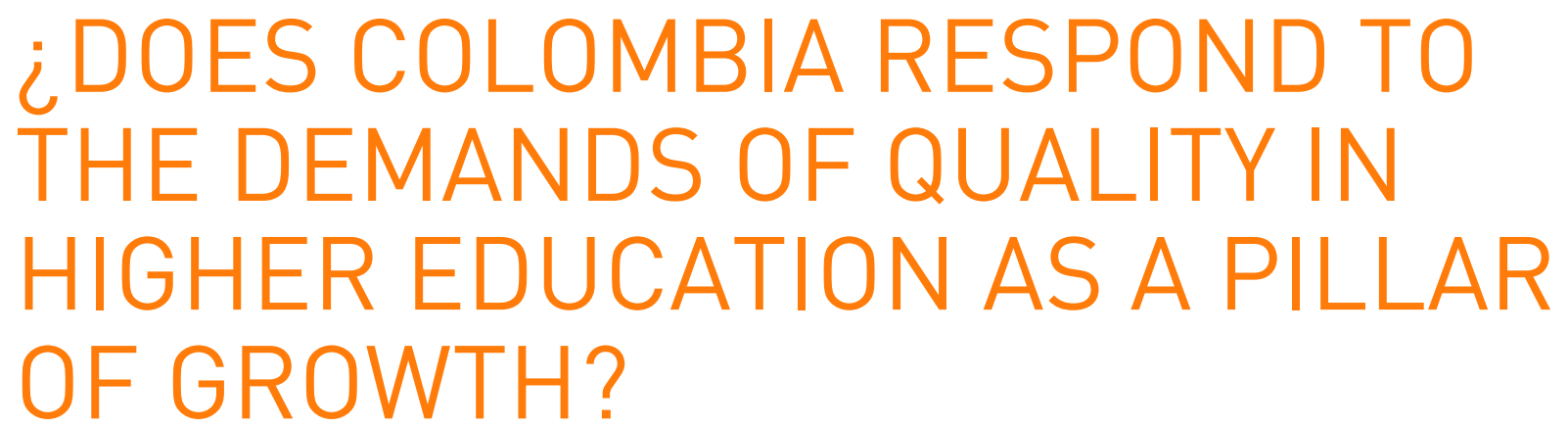

\section{Cecilia Garzón Daza ${ }^{1}$}

Fundación San Mateo - Universidad Católica de Colombia.

\section{RESUMEN}

Dentro del ámbito universitario la calidad ha sido vista con la rigurosidad que la misma requiere, debido a que se ha consolidado como la respuesta positiva a los procesos de acreditación de instituciones y programas universitarios. Razón por la cual, la calidad en la educación superior debe continuar su consolidación en el ámbito

$1 \quad$ Post doctora en Educación de la Universidad Santo Tomás. Doctora en Ciencias de la educación de la Universidad Pedagógica y Tecnológica de Colombia - U. P. T. C. Docente investigadora de la Fundación San Mateo y Directora de Calidad Universidad Católica de Colombia. universitario con miras a demostrar la capacidad de gestión de la institución universitaria, en pro de favorecer esta instancia en todos los aspectos; en donde, la gestión del conocimiento, investigación e internacionalización van a hacer la respuesta positiva para que Colombia afiance su posicionamiento frente a sus homologas en la región.

Así, el presente artículo de revisión se estructura, a partir del marco normativo que consolida la calidad en la educación superior, 
seguido de las experiencias significativas en la materia, haciendo alusión a la acreditación tanto nacional como internacional, siendo relevante la presencia del programa de ingenierías en el MERCOSUR y finalmente haciendo una clara referencia a la responsabilidad que tiene el país en continuar con este proceso.

PALABRAS CLAVE: educación superior, calidad, acreditación, investigación, internacionalización, sociedad del conocimiento

\section{ABSTRACT:}

Within the university context, quality has been viewed with the rigor that it requires, because it has been consolidated as the positive response to the accreditation processes of institutions and university programs. Reason why, the quality in the superior education must continue its consolidation in the university scope with a view to demonstrate the capacity of management of the university institution, in favor of favoring this instance in all aspects; where, knowledge management, research and internationalization are going to make a positive response so that Colombia consolidates its position in relation to its counterparts in the region.

Thus, this review article is structured, based on the normative framework that consolidates quality in higher education, followed by significant experiences in the subject, alluding to both national and international accreditation, the presence of the engineering in MERCOSUR and finally making a clear reference to the responsibility that the country has to continue with this process.

KEYWORDS: higher education, quality, accreditation, research, internationalization, knowledge society

\section{INTRODUCCIÓN}

Uno de los fundamentos de la educación superior es atender las exigencias del mundo actual, en materia de calidad educativa a través de la acreditación universitaria que se da siempre y cuando medie un proceso de calidad. Para lo cual se debe hacer referencia al sólido marco normativo existente, que ha permitido consolidar el sistema de gestión de la calidad respaldado por los diferentes entes que tienen estrecha relación con el tema, teniendo como punto de partida el Ministerio de Educación Nacional. En este ámbito, las universidades tienen un papel protagónico y por ello merecen ser estudiadas.

Así, desde que la universidad se originó ha tenido una clara responsabilidad en generar conocimiento, el cual se ha ido transformando conforme el tiempo ha ido avanzando, integrando elementos que la hacen día a día más dinámica, compleja, centro de la investigación y desarrollo. En cuyo caso, la calidad constituye la base piramidal para que la universidad logré cumplir las exigencias del mundo actual permitiendo identificar algunos retos importantes para su implementación y consolidación.

De ahí que, la calidad en la actualidad no es un tema exclusivo del sector empresarial y de las políticas de Estado, sino que hace parte fundamental de la academia, haciendo que ésta sea el centro del cual emergen las respuestas para ascender, posicionarse y conservar una educación superior de calidad.

En este orden de ideas, debe existir desde la educación superior una clara definición del papel o rol que juega la acreditación dentro del marco de la gestión de la calidad, cuya visión debe perpetuarse en el tiempo por medio de un accionar constante que lleve a la educación superior colombiana a ser un ejemplo en los países de la región; toda vez, que la calidad es una prioridad y no debe ser tomada a la ligera, máximo cuando la norma la define, promueve y 
consolida los espacios para su aplicación.

Razón por la cual, el presente artículo de revisión se estructura, a partir del marco normativo que consolida la calidad en la educación superior, seguido de las experiencias significativas en la materia, haciendo alusión a la acreditación tanto nacional como internacional, siendo relevante la presencia del programa de ingenierías en el MERCOSUR y finalmente se establece una clara referencia a la responsabilidad que tiene el país en continuar con este proceso.

Por último, la metodología propuesta parte de un estudio descriptivo que procura "brindar una buena percepción del funcionamiento de un fenómeno y de las maneras en que se comportan los factores o elementos que lo componen. Los estudios descriptivos llegan finalmente a conclusiones generales construidas por medio de abstracciones, que dan cuenta de los hechos observados" (Hernández, 2010, pág. 97), acompañada de una técnica documental, la cual se orienta a establecer contacto directo con la fuente de información con relación al tema en estudio.

\section{UN ACERTADO MARCO NORMATIVO EN PRO DE LA CALIDAD DE LA EDUCACIÓN SUPERIOR COLOMBIANA}

Colombia ha sido recurrente en el deseo por lograr afianzar la calidad de la Educación superior; por ello, no ha restringido los esfuerzos en lograr una regulación clara y precisa que ofrezca los lineamientos en la materia, tema que constituye el punto central del presente apartado.

La Ley 30 de 1992 ordenó la creación y funcionamiento del Consejo Nacional de Educación Superior - CESU - a través del artículo 34, definiendo sus funciones en el artículo 36, siendo una de estas los mecanismos para evaluar la calidad académica de las instituciones de Educación Superior y de sus programas. De igual manera en la Ley 30 de 1992 en los artículos 53, 54, 55 y 56 se crea Sistema Nacional de Acreditación - SNA - y su sistema de autoevaluación e información. Así, en el artículo 53 hace referencia a que es voluntario de las instituciones de educación superior acogerse al sistema de acreditación; estableciendo además el carácter temporal de ésta y en el artículo 56 se establece la creación del Sistema Nacional de Información de la Educación Superior - SNIES - que tendrá como objetivo fundamental divulgar la información para orientar a la comunidad sobre la calidad, cantidad y características de las instituciones y programas del Sistema.

De tal modo, que la acreditación tendrá carácter temporal. Las instituciones que se acrediten disfrutarán de las prerrogativas que para ellas establezca la ley y las que señale el Consejo Superior de Educación Superior - CESU -. La ley definió la autoevaluación institucional como una tarea permanente de las instituciones de Educación Superior y hará parte del proceso de acreditación.

Igualmente, con la creación del SNIES cuyo objetivo es divulgar información para orientar a la comunidad sobre la calidad, cantidad y características de las instituciones y programas del Sistema, se logró organizar de forma permanente la información relevante sobre la educación superior. La reglamentación SNIES corresponde al Consejo Nacional de Educación Superior.

Por su parte, el Sistema Nacional de Acreditación - SNA - según el Decreto 2904 de 1994 y el Acuerdo 04 de 1995 está integrado por:

- El Consejo Nacional de Educación Superior. Este organismo tiene una naturaleza académica, integrado por siete miembros, designados para periodos de cinco años por el CESU, personas de las más altas calidades científicas y profesionales, con prestancia 
nacional e internacional. Dicho Consejo redefine su intencionalidad, con el Acuerdo No. 02 de 2005 del CESU, por medio del cual modifica el reglamento, integración y las funciones del Consejo Nacional de Acreditación.

- El Consejo Nacional de Acreditación - CNA - integrado, entre otros, por las comunidades académicas y científicas. Su objetivo primordial es promover y ejecutar la política de acreditación adoptada por el CESU, a través de la coordinación de los respectivos procesos hasta lograr la evaluación final, que debe ser remitida al Ministerio de Educación Nacional.

- El proceso de acreditación que es de naturaleza mixta, son promulgados por el Ministro de Educación Nacional, cuyo proceso es dirigido por las mismas instituciones, por los pares académicos y por el CNA, conformado por académicos en ejercicio, pertenecientes a las distintas IES.

- La comunidad académica, que contribuye $o$ hace parte al proceso de acreditación

El Sistema Nacional de Acreditación se inscribe en el concepto de fomento de la calidad de la Educación, como mecanismo para la búsqueda permanente de los más altos niveles de calidad por parte de las instituciones de educación superior que se acojan al sistema.

El proceso de acreditación, de acuerdo con la experiencia lograda por el sistema a través de más de diez años de experiencia y de la identificación con "sistemas maduros de aseguramiento de la calidad de diversos países, se soporta en una sólida cultura de la autoevaluación, la evaluación por pares externos, la evaluación síntesis hecha por el CNA y en el acto de acreditación proferido por el MEN" (Mejía, P y Duque, S, 2013, pág. 16); de ahí que, el concepto de calidad que utiliza el CNA es multidimensional e integrado. Los criterios sobre los cuales opera el Sistema Nacional de Acreditación son: coherencia, eficacia, eficiencia, equidad, integridad, pertinencia, sostenibilidad, responsabilidad y universalidad.

Estos criterios son factores valorativos que contribuyen en el alcance de la calidad, tanto de la institución como del programa académico. Por su parte, la evaluación de la calidad en el campo de la acreditación implica un ejercicio complejo que, a pesar de apoyarse en indicadores cuantitativos y objetivos, no puede renunciar a su carácter cualitativo y hermenéutico. "Este proceso ha estado muy ligado desde sus inicios, a la idea de la autonomía y la autorregulación, complementada con la exigencia de rendición de cuentas que se hace a la Educación Superior desde distintos sectores sociales" (Mejía, P y Duque, S, 2013, pág. 17).

La acreditación es la respuesta a la necesidad derivada de múltiples escenarios, en pro de fortalecer la calidad de la Educación Superior y al propósito de hacer reconocimiento público del logro de altos niveles de calidad. Se presenta además en un momento crítico como respuesta a los imperativos del mundo moderno, que otorgan un carácter central a la calidad de la Educación Superior como medio de desarrollo del país.

En Colombia, el proceso de acreditación no se origina del marco de la inspección y la vigilancia del Estado, sino del fomento, reconocimiento y mejoramiento continuo de la calidad.

De otra parte, el gobierno nacional creó la Comisión Consultiva de Educación Superior, mediante los Decretos 1176 de 1999 que transformó el CESU y el Decreto 2320 de 1999 que regula su funcionamiento; este organismo estuvo encargado de asesorar al Ministro de Educación acerca de las solicitudes de nuevas personerías jurídicas, apertura de seccionales, cambio de carácter académico; entre otros aspectos. 
Otro actor del sistema de calidad es la Comisión Nacional Intersectorial de Aseguramiento de la Calidad de la Educación Superior -CONACES -, creado por el Decreto 2230 de 2003, a través del artículo 37 de conformidad con el artículo 45 de la Ley 489 de 1998. Con la creación del CONACES, el gobierno nacional suprime la Comisión Consultiva de Educación Superior y la Comisión Nacional de Doctorados y Maestrías, y sus funciones las asume el CONACES.

Posteriormente mediante la Ley 1188 de 2008 se asignaron funciones de evaluación de la calidad al CONACES, las cuales son: coordinación y orientación del aseguramiento de la calidad de la educación superior, evaluación del cumplimiento de los requisitos para la creación de instituciones, su transformación y redefinición, sus programas académicos y demás funciones que le sean asignadas. CONACES posteriormente fue reglamentada por el Decreto 5012 de 2009 y modificada por la Resolución 5290 de 2012.

En consonancia con lo señalado hasta ahora, se encuentra la modernización de los lineamientos de acreditación y el concepto de calidad promovido por el CESU, a través del Acuerdo 01 de 2018. Así, según los lineamientos iniciales la calidad en la educación superior hacía referencia a:

La síntesis de características que permiten reconocer un programa académico específico o una institución de determinado tipo y hacer un juicio sobre la distancia relativa entre el modo como en esa institución o en ese programa académico se presta dicho servicio y el óptimo que corresponde a su naturaleza (Consejo Nacional de Educación Superior [CESU], 2018).

Con los nuevos lineamientos se habla de calidad como la síntesis "de condiciones que, teniendo en cuenta la identidad institucional, permiten evaluar, en un tiempo determinado, cómo las instituciones y los programas logran los resultados de aprendizaje de los estudiantes y contribuyen con el desarrollo de los territorios y el país" (Consejo Nacional de Educación Superior [CESU], 2018).

Se puede observar que el cambio conceptual contribuye a albergar diferentes actores que incluye el sector socio - económico del país; mientras que la primera hacía referencia a procesos estrictamente internos de la universidad; esto obedecía a que para el año 1992 no se pensaba en la globalización del conocimiento, no se tenía claridad hasta dónde los avances tecnológicos iban a llegar y, por ende, no había un reconocimiento de la sociedad del conocimiento. Razón por la cual, el proceso era estrictamente interno.

Por último, se encuentra el Decreto 1075 de 2015 que se erige como la norma marco del sector educativo que integran todos los postulados antes señalados en materia de calidad y acreditación. Con este decreto se ha dado un importante avance en el proceso de sistematización del sector educativo en sus diferentes niveles, permitiendo que el sector cuente con una propuesta de funcionamiento moderna y actualizada.

\section{EXPERIENCIAS SIGNIFICATIVAS DE LA EDUCACIÓN SUPERIOR COLOMBIANA EN MATERIA DE CALIDAD}

La revisión del marco normativo permitió identificar los avances que Colombia ha tenido en materia de calidad educativa, dejando claro, que ésta ha sido una preocupación constante para el Estado colombiano, en pro de optimizar los procesos actuales, atendiendo las exigencias del mundo actual en la materia.

La educación superior es transcendental para el éxito y prosperidad de una nación. Día a día existe más evidencia del impacto positivo por lograr niveles más altos de estudios, "en 
un amplio rango de resultados sociales y económicos, desde el aumento de ingresos y productividad hasta el compromiso político y la igualdad social" (Comisión Económica para América Latina y el Caribe [ CEPAL], 2000, pág. 78). La importancia cada vez mayor de la educación superior se ve reflejada en la demanda creciente, especialmente en economías emergentes como la de Colombia, donde las competencias requeridas evolucionan con rapidez.

En la última década, la tasa neta de matriculados en educación superior en Colombia es cercano al " $50 \%$, el doble que hace una década, y el $21 \%$ de las personas entre 25 y 64 años ha alcanzado niveles de educación superior" (Organizaciones de las Naciones UUnidas para la Educación, la Ciencia y la Cultura, 2013, pág. 160). De estos, cerca de la mitad se han graduado de programas de licenciatura o su equivalente, mientras que el resto han alcanzado títulos de programas técnicos superiores más cortos. De tal modo, que la calidad va más allá de ser una simple expansión de la educación; así, incluye no solo logros, sino condiciones y procesos, no necesariamente en el ámbito académico, sino sicosociales y ciudadanos, siendo los dos últimos los que requieren ser más afianzados.

Se tiene entonces, que los procesos actuales de modernización están en gran medida determinados por la emergencia de la sociedad del conocimiento, que debe ser vista, no solamente como una transformación social, sino como una tendencia en crecimiento que exige universidades más competitivas con programas académicos acordes a las exigencias del mundo actual, que permitan el dinamismo en el mundo global; en donde, la estructura ocupacional cada vez exige niveles más altos de preparación. Ante estos cambios, "han debido redefinírselos espacios institucionales de la sociedad moderna para la producción y reproducción de sus conocimientos más elaborado: las universidades" (Organizaciones de las Naciones UUnidas para la Educación, la Ciencia y la Cultura, 2013, pág. 131).

Comúnmente las universidades monopolizaban las funciones de formación, en cuanto a "recursos humanos calificados, producción de conocimiento avanzado, ciencia, humanidades, artes, investigación mediados entre lo público y lo privado, funciones que dados los avances tecnológicos han exigido su transformación" (Iriarte, 2015, pág. 108), a la luz, de una mayor apertura social para dar respuesta a la creciente demanda por movilidad social con ocasión de los estudios. Situación que exigió cambios sustanciales para estar a la altura de las exigencias; en donde, se tuvo que dar paso a transformaciones de fondo que le han permitido a la educación superior mantenerse vigente, con la ampliación de programas, opciones de crédito para las matrículas, apoyo en investigación, entre otras, le han llevado a ser líder en calidad; gracias, además a que sus procesos están acompañados de mayor investigación científica $y$ al predominio de la oferta en posgrados.

Entonces, en el ámbito de las políticas para la educación superior, la acreditación y aseguramiento de la calidad ha ido tomando un mayor auge, siendo esta la experiencia significativa; debido a que "la acreditación institucional se centra en el cumplimiento de los postulados de la misión y el proyecto institucional; en la pertinencia social, cultural y pedagógica; a la manera como la institución afronta el cumplimiento de sus funciones básicas" (Consejo Nacional de Acreditación [CNA], 2018, pág. 11) en los distintos campos de acción de la educación superior, al clima institucional, a los recursos con que cuenta y su desempeño global. En este orden de ideas, Colombia ha logrado un avance memorable en la materia, debido a que en la actualidad son 51 universidades entre públicas y privadas que han logrado tan alta distinción. 


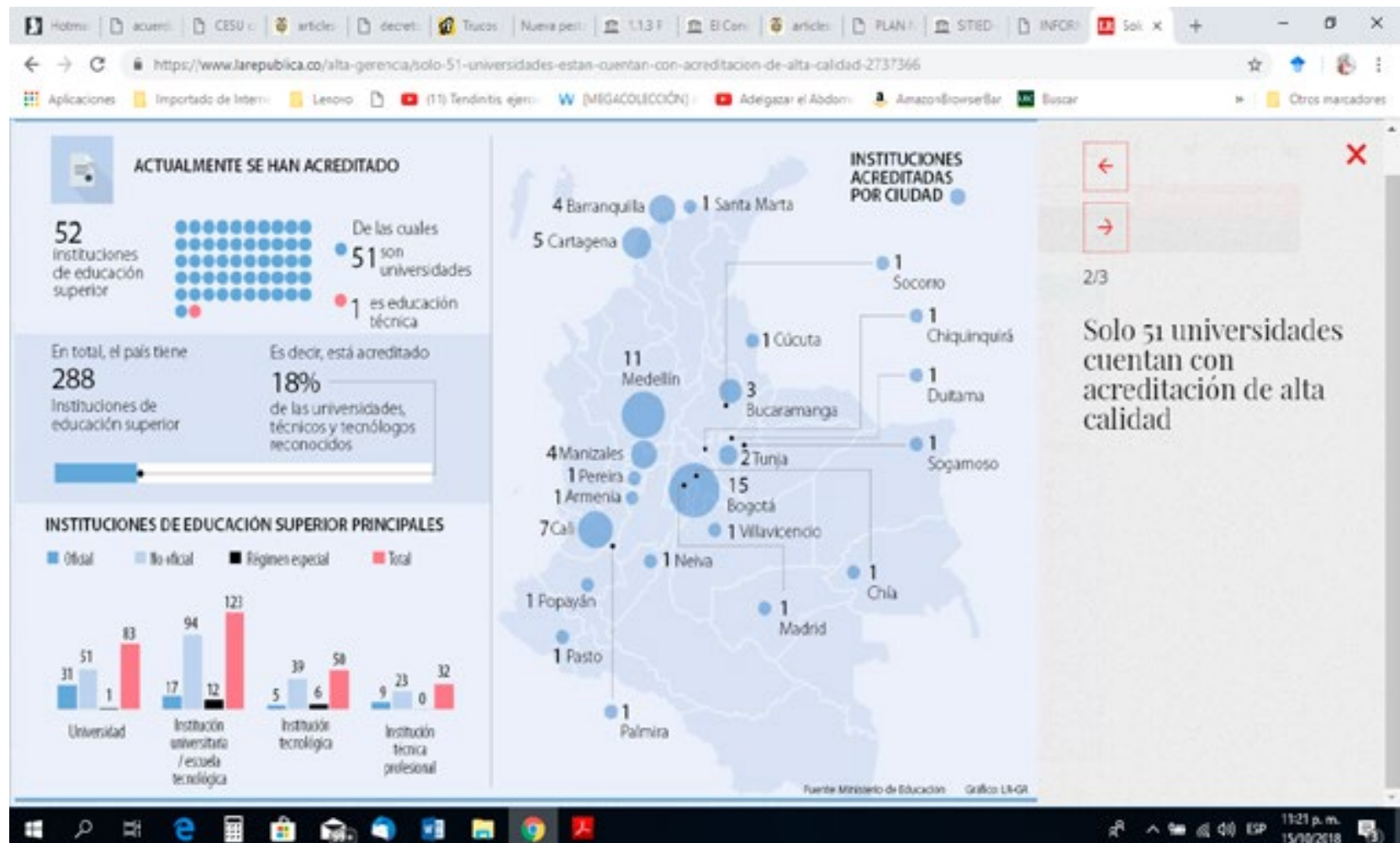

Figura 1. Universidades acreditadas en

Colombia

Fuente: Ministerio de Educación (2017)

Situación que ha llevado a que Colombia sea vista de forma diferente, lo que le ha permitido afianzar la política de internacionalización de la educación superior; esto debido a que, desde el 2010 el CNA ha participado activamente en los procesos de reconocimiento de buenas prácticas de aseguramiento de la calidad, recibiendo en el año 2012 la primera certificación sobre alineamiento del Consejo a las Directrices de Buenas Prácticas, establecidas por la Red Internacional de Agencias de Aseguramiento de la Calidad en la Educación Superior - INQAAHE -, con una vigencia de cinco años, generando un compromiso de gestión y sostenibilidad en calidad, con el fin de tener una permanencia activa que contribuya a que la educación superior colombiana sea compatible con la globalización del conocimiento, y en la región logré a futuro una mejor posición.
Lo anterior, teniendo en cuenta que el proceso de acreditación institucional actualmente no es obligatorio, y que su obligatoriedad exigiría una serie de ajustes a los criterios actuales, los cuales deberían, integrar la vinculación de empleadores y empresas en el desarrollo de nuevos procesos y estándares consolidados de aseguramiento de la calidad, beneficiando el mejoramiento en el desempeño institucional, que se orienta a aumentar la oferta de programas de posgrado y promover el gasto en investigación y desarrollo.

De ahí que, la iniciativa más reciente del gobierno colombiano para mejorar el desempeño institucional ha sido el adoptado en el 2015 y conocido como el Modelo de Indicadores de Desempeño de la Educación - MIDE -, el cual debería estimular la competencia entre las instituciones a fin de mejorar el desempeño. 
"El sistema de clasificación MIDE es un instrumento potencialmente útil para incentivar más concentración y transparencia en torno a la calidad de la educación superior" (Ministerio de Educación Nacional, 2016, pág. 309), el cual requiere ser más explorado y tecnificado para que de los resultados esperados, en especial, los orientados a evaluar los procesos de acreditación.

En suma, la experiencia significativa con relación a la calidad de la educación superior se centra en los procesos de acreditación de las universidades, fundamentado en la voluntad que se tenga para lograr tal distinción; evidencia clara de hacer instituciones de calidad. Aspecto que exige que día a día la universidad sea pensada desde la adquisición constante de recursos económicos y humanos, que le permita ampliar la financiación disponible para estudiantes, investigación e internacionalización afianzando el crecimiento y por ende la calidad.

\section{¿QUÉ TENEMOS, HACIA DÓNDE VAMOS Y CÓMO LOGRAR AFIANZAR LA CALIDAD EN LA EDUCACIÓN SUPERIOR COLOMBIANA?}

Las apreciaciones señalados en los apartados anteriores, son muestra clara, de los esfuerzos realizados por Colombia para posicionar la educación superior con calidad, generando procesos transversales que contribuyen ostensiblemente a tal propósito; por eso, claramente se puede decir que tenemos un marco normativo sólido, evidencia clara de la preocupación constante de quienes tienen en sus manos la ardua laboral de afianzar los cimientos para que la educación superior tenga como articular todos sus procesos. Reflejo de ello, es el informe presentado por el Ministerio de Educación (2017) sobre calidad y equidad 2016 - 2026, a través del cual se hace un diagnóstico desde cinco puntos: acceso y cobertura, permanencia, calidad, pertinencia y financiación.
Así, continuando con el tema en discusión es importante señalar que la consolidación del sistema de aseguramiento de la calidad ha permitido elevar el nivel de las instituciones y programas académicos, en cuyo propósito, se destaca la acreditación en alta calidad tanto de programas como de Instituciones de Educación Superior - IES, lo que permite plantear hacía dónde vamos, que claramente es afianzar el sistema de aseguramiento de la calidad que debe atender los objetivos propuestos por los países que pertenecen a la Organización para la Cooperación y el Desarrollo Económicos OCDE - (2016) que hacen referencia a:

- Promover la equidad, pertinencia y eficiencia

- Garantizar estándares mínimos, a fin de proteger los intereses de cada estudiante

- Promover una cultura de calidad y profesionalismo que lleve al mejoramiento continuo en el sistema más amplio de educación superior y en el propio proceso de aseguramiento de calidad

- Apoyar la vinculación activa de las partes interesadas, especialmente estudiantes y empleadores, a fin de promover la capacidad de respuesta y pertinencia de la educación superior;

- Permitir la diversidad de instituciones, programas y modos de prestación de educación superior, para promover su flexibilidad y su adecuación a un fin específico;

- Incorporar transparencia y accesibilidad para inspirar seguridad y confianza

Objetivos que se unen con lo expuesto por Brunner, J y Villalobos, C (2014) cuando hace referencia a lo señalado por la Organización de Estados Iberoamericanos para la Educación, la Ciencia y la Cultura - OEI - en el informe Metas Educativas 2021 (2008): 
El desarrollo de las naciones depende ahora más que nunca de la calidad de la formación a la que se accede en las universidades y del conocimiento que se pueda generar y acumular en ellas. El estado de los países de la región iberoamericana es, en este sentido, muy débil, y los pone en desventaja y en riesgo de exclusión respecto del progreso acelerado del mundo desarrollado, porque los avances tecnológicos generan dinámicas de exclusión aún mayores que las tradicionales (Brunner, J y Villalobos, C, 2014, pág. 10).

De tal modo, que Colombia debe continuar en la consecución de los objetivos antes señalados; aspecto en el cual los esfuerzos deben ser conjuntos entre las diferentes entidades que tienen estrecha relación con optimizar la calidad en la educación superior; especialmente en lo concerniente a la consecución de recursos para la investigación e internacionalización. Situación que se debe dar, atendiendo la "transición hacia la economía del conocimiento, la cual ha ocasionado transformaciones profundas en las estructuras productivas y una redefinición de las funciones de las instituciones de educación superior" (Brunner, J y Villalobos, C, 2014, pág. 12).

Esto respondería claramente al papel de las universidades en la sociedad del conocimiento, equiparándose a los cambios en las dinámicas y las relaciones de las instituciones de educación superior con el sector productivo, tal como lo han hecho los países desarrollados. Razón por la cual, la transferencia de tecnología y conocimiento por medio de la aplicación y diseminación de los resultados generados de la investigación académica y la creación de beneficios económicos derivados de estos, serían una constante en la educación superior colombiana, afianzando los objetivos de la calidad. Planteamientos que permiten abordar, la última pregunta del título planteado del presente apartado: cómo lograr afianzar la calidad.
Para tal fin, se deben fortalecer y enriquecer programas como "Colombia Científica" promovido por el Ministerio de Educación Nacional (2017) en pro de mejorar la calidad de la educación superior, teniendo como eje el fortalecimiento de la docencia, la investigación y la internacionalización de las instituciones educativas, ha sido un desafío para el país en materia educativa. De modo tal, que:

"Colombia Científica" le apunta al cierre de brechas en el acceso de los jóvenes a la educación superior de calidad, generando relaciones entre IES acreditadas con aquellas no acreditadas, en procesos de formación de alto nivel y programas de Investigación, Desarrollo Tecnológico e Innovación $(I+D+i)$ que impulsen el desarrollo regional y respondan a los retos del desarrollo social y productivo del país (Ministerio de Educación Nacional [MEN], 2017b, pág. 1).

Como se puede observar existe un claro compromiso por parte del Ministerio de Educación Nacional en fortalecer la calidad de la educación superior; a través de programas que se equiparán con las exigencias del mundo global; haciendoénfasis que "Colombia científica" también integra el "Ecosistema científico" y el "Pasaporte científico", siendo la orientación del primero contribuir "al mejoramiento de la calidad de las IES colombianas participantes, a partir de la conformación de alianzas que impulsen el desarrollo regional y que respondan a los retos del desarrollo social y productivo del país" (Ministerio de Educación Nacional [MEN], 2017b, pág. 1); y del segundo, busca brindar oportunidades a los "mejores profesionales, docentes e investigadores del país la oportunidad de efectuar estudios de maestría y doctorado en las mejores instituciones del mundo" (Ministerio de Educación Nacional [MEN], 2017b, pág. 1).

El desarrollo de estos programas unidos a los objetivos propuestos en la Agenda 2021 tendrán 
como resultado que la acreditación de las IES sea un propósito en consenso y no la decisión de algunas instituciones; debido a que, dicha acreditación posicionará a Colombia en la región como uno de los mejores países en materia de calidad educativa y así, podrá acceder a la acreditación internacional del MERCOSUR EDUCATIVO, siendo un claro ejemplo de ello, la respuesta positiva que tuvo Colombia, frente a la Convocatoria de Ingeniería 2010, por parte del Sistema de Acreditación Regional de Carreras Universitarias - ARCUSUR -, siendo acreditadas: "Ingeniería Química de la Universidad Nacional de Colombia, sedes Bogotá, Medellín y Manizales; Ingeniería Civil de la Escuela Colombiana de Ingeniería Julio Garavito e Ingeniería Eléctrica de la Universidad del Valle" (Consejo Nacional de Acreditación [CNA], 2010, pág. 1).

Este tipo de experiencias de acreditación internacional se encuentran avaladas por los gobiernos de los países pertenecientes al MERCOSUR - Argentina, Brasil, Chile, Bolivia, Colombia, Paraguay, Uruguay y Venezuela -, siendo las únicas acreditaciones que pueden apoyar el reconocimiento de títulos para facilitar la movilidad académica de los profesionales. Acreditación que debe ser lograda por todas las áreas del conocimiento y disciplinas que merecen tener dicho reconocimiento; asi afianzar la calidad, requiere avanzar en el desarrollo de propuestas, programas, estrategias y políticas que apunten a que la calidad continúe siendo el eje transversal de la educación superior.

\section{CONCLUSIONES}

El artículo desarrollado permite concluir que desde la norma existe un gran avance en lo que a calidad se refiere; a través de la cual se establecen, no solamente, los lineamientos sino los objetivos propuestos y acciones a seguir, acompañada del compromiso que tiene Colombia como Estado parte de la normatividad que hace referencia a la calidad y miembro de comunidades internacionales. En este sentido, la norma debe continuar en ascenso atendiendo las exigencias de la sociedad del conocimiento; por eso, es necesario que se transforme como la tecnología lo hace a diario; esto evitará procesos anquilosados que lleve a que las instituciones de educación superior se equiparen a sus homologas en la región y que el país tome mayor distancia en materia educativa frente a los países desarrollados.

Se puede afirmar, entonces, que como vamos, vamos bien gracias a que el Estado colombiano ha comprendido la necesidad de estar al día en materia normativa como orientadora de los lineamientos de la calidad en la educación superior. En este orden de ideas, solamente resta consolidar políticas públicas que apunten a generar las líneas de acción para afianzar los objetivos propuestos en la materia; afianzar la Agenda de las instituciones en el logro de los proyectos, programas y recursos que den vía libre para que temas como la investigación e internacionalización sean ejemplos insignes de crecimiento y, por ende, reflejo de procesos de gestión en calidad.

Acreditarse seguirá siendo la meta para las universidades colombianas, en pro de optimizar sus procesos y ser vistas en la comunidad académica global como una educación competitiva, reflejo de procesos hilados y transformadores que se centran en el desarrollo, ejecución y continuidad de proyectos como "Colombia científica" que ponen de manifiesto el compromiso del país en el interés por la calidad 
educativa; aunado a procesos de acreditación internacional como las ofrecidas por el MERCOSUR, siendo un ejemplo las Ingenierías, que en un futuro no muy lejano, deben extenderse a todas las áreas del conocimiento; momento en el cual la pluralidad de profesiones en ciencias administrativas, económicas y financieras; en ciencias sociales y humanidades, en ciencias exactas e ingenierías, entre otras serán muestra clara de calidad al lograr la acreditación no solamente a nivel nacional sino internacional.

Por ello, se debe continuar desarrollando un trabajo conjunto entre las diferentes entidades para que se den procesos sólidos y viables que apunten a la calidad; para ello, el recurso humano, tecnológico, económico y político debe estar presente formando un engranaje perfecto que permita que todo el accionar en materia de calidad se consolide convirtiéndose en una respuesta positiva y en futuro cercano Colombia sea reconocida por su alto nivel de participación en redes internacionales de investigación, movilidad estudiantil y la internacionalización de estudiantes y docentes.

Esto redundará en respuestas positivas en materia de equidad, bienestar y ambiente institucional $e$ inclusive en procesos de proyección social; pues desde este punto de vista, y como se expuso en el desarrollo del artículo la calidad tiene una función transversal en toda la educación superior; es decir sí hay calidad, temas como cobertura y pertinencia serán cosa del pasado. Resulta claro, entonces que la calidad constituye la base piramidal de la educación superior.

\section{REFERENCIAS}

Brunner, J y Villalobos, C. (2014). Politicas de Educación Superior en Iberoamérica 2009 - 2013. Santiago de Chile: Universidad Diego Portales.

Comisión Económica para América Latina y el Caribe [ CEPAL]. (2000). Equidad, desarrollo y ciudadanía. New York: CEPAL.

Congreso de la República de Colombia. (29 de diciembre de 1998). Ley 489. Por la cual se dictan normas sobre la organización y funcionamiento de las entidades del orden nacional, se expiden las disposiciones, principios $y$ reglas generales para el ejercicio de las atribuciones previstas en la Constitución Nacional. Bogotá, D.C.

Congreso de la Repúbliica de Colombia. (25 de abril de 2008). Ley 1188. Por la cual se regula el registro calificado de programas de educación superior y se dictan otras disposiciones. Bogotá, D.C.

Consejo Nacional de Acreditación [CNA]. (30 de noviembre de 2010). Colombia participa enn procesos de acreditación en el MERCOSUR. Obtenido de https://www. cna.gov.co/1741/article-262257.html

Consejo Nacional de Acreditación [CNA]. (2018). Informe de gestión 2017. Bogotá, D.C: CNA.

Consejo Nacional de Educación Superior CESU -. (14 de diciembre de 1995). Acuerdo No. 6. Por el cual se adoptan las políticas generales de acreditación $y$ se derogan las normas que sean contrarias. Bogotá, D.C.

Consejo Nacional de Educación Superior [CESU]. (23 de junio de 2005). Por e 
cual se subroga el acuerdo 1 de 2000 del Consejo Nacional de Educación Superior - CESU -, y con el cual se expide el reglalmento, se determina la integración y las funciones del Consejo Nacional de Acreditación. Bogotá, D.C.

Consejo Nacional de Educación Superior [CESU]. (11 de agosto de 2018). Acuerdo 01. Por el cual se actualizan los lineamientos para acreditación de alta calidad institucional y de programas de pregrado. Bogotá, D.C.

Hernández, S. (2010). Metodología de la Investigación (5 ed.). Cánada: McGraw Hill.

Iriarte, C. (2015). La definición de prioridades como componentes del planteamiento en ciencia y tecnología para la universidad: el caso de la Universidad Nacional Autónoma de Honduras. Revista Cubana de Educación Superior, 107 - 129.

Mejía, P y Duque, S. (29 de enero de 2013). Sistema de aseguramiento de la calidad para la educación superior: una tarea pendiente. Obtenido de https://www.mineducacion.gov.co/1759/ articles-341917_archivo_pdf.pdf

Ministerio de Educación Nacional [MEN]. (31 de marzo de 2017b). Obtenido de https:// www.mineducacion.gov.co/1759/w3article-360383.html?_noredirect=1

Ministerio de Educación Nacional. (28 de diciembre de 1992). Ley 30. Por la cual se organiza el servicio público de la educación superior. Bogotá, D.C.

Ministerio de Educación Nacional. (28 de diciembre de 2009). Decreto 5012. Por el cual se modifica la estructura del Ministerio de Educación Nacional, $y$ se determinan las funciones de sus dependencias. Bogotá, D.C.

Ministerio de Educación Nacional. (24 de mayo de 2012). Resolución 5290. Por el cual se reorganiza la Comisión Nacional Intersectorial de Aseguramiento de la Calidad de la Educación Superior y se dictan otras disposiciones. Bogotá, D.C.

Ministerio de Educación Nacional. (26 de mayo de 2015). Decreto 1075. Por medio del cual se expide el Decreto Único Reglamentario del Sector Educación. Bogotá, D.C.

Ministerio de Educación Nacional. (2016). Revisión de políticas nacionales de educación. La educación en Colombia. Colombia: MEN.

Ministerio de Educación Nacional. (2017a). Plan Nacional Decenal de Educación 2016 - 2026. El camino hacia la calidad y la equidad. Bogotá, D.C: MEN.

Organización de los Estados Iberoamericanos [OEI]. (2008). Metas Educativas 2021. La educación que queremos para la generación de los Bicentenarios. Madrid: OEI.

Organización para la Cooperación y el Desarrollo Económicos [OCDE]. (2016). Revisión de política nacionales de educación. La educación en Colombia. Paris: OCDE.

Organizaciones de las Naciones UUnidas para la Educación, la Ciencia y la Cultura. (2013). Situación educativa de América Latina y el Caribe: hacia la educación de calidad para todos al 2015. Santiago de Chile: UNESCO.

Presidencia de la República. (31 de diciembre de 1994). Decreto 2904. Por el cual se reglamenta los artículo 53 y 54 de la Ley 
30 de 1992. Bogotá, D.C.

Presidencia de la República. (29 de junio de 1999). Decreto 1176. Por el cual se transforma el Consejo Nacional de Educación superior, CESU, se fusionan los Comités Asesores de que trata el Capítulo III de la Ley 30 de 1992 y se dictan otras disposiciones. Bogotá, D.C.

Presidencia de la República. (23 de noviembre de 1999). Decreto 2320. Por el cual se regulan la integración yel funcionamiento de la Comisión Consultiva de Instituciones de Educación Superior y se dictan otras disposiciones. Bogotá, D.C.

Presidencia de la República. (8 de agosto de 2003). Decreto 2230. Por el cual se modifica la estructura del Ministerio de Educación Nacional, y se dictan otras disposiciones. Bogotá, D.C. 